\title{
Nonlinear Transmission characteristics of Eriochrome Cyanine R Dye Chromophore Under CW He-Cd Laser Illumination
}

\author{
Monica S \\ Department of Physics, Fatima Mata National College, Kollam, India
}

Available online at: www.isroset.org

Accepted: 26/Jul/2018, Online: 31/Aug/2018

\begin{abstract}
The nonlinear optical limiting behavior of an organic dye, Eriochrome Cyanine $\mathrm{R}$ in aqueous solution, was investigated under excitation with $\mathrm{cw}$ He-Cd laser light at $442 \mathrm{~nm}$. The nonlinear optical responses of the material were studied, using single-beam Z-scan technique. The optical limiting behavior of the dye chromophore is also demonstrated.
\end{abstract}

Keywords- Eriochrome Cyanine R; Z-scan; Optical Limiting

\section{INTRODUCTION}

The rapid technological developments that took place in Optics during the last few decades have led to the search for materials with large optical nonlinearities, which would also satisfy all the technological requirements such as wide active optical frequency range, fast optical response time, high damage threshold, adaptability and capability to interfacing with other materials etc. Nonlinear optical (NLO) materials have great impact on information technology, industry, life sciences, and invariably in defense technology. Most of the current investigation made in nonlinear optical materials is motivated by its potential for high performance applications which are not accessible by electronics. Tremendous advance have been made in this field in a short period, driven by the current need for faster and higher means of communications and data processing and its potential in realizing all optical systems. One of the important applications of these materials is in optical limiting (OL) devices used to protect eyes and sensors against damage by exposure to sudden high-intensity light, which still remains a challenging problem. Optical limiters can transmit lowintensity laser pulses effectively and attenuate high-intensity laser pulses strongly by using the NLO properties of the materials [1]. The concept of optical limiting is based on the ability of an optical limiter to reduce the intensity of the incoming light to safe (low) intensities . An optical limiting process comprises an effective suppression of potentially damaging high intensity radiation, while permitting efficient transmission at normal light intensities, and hence can attenuate intense light bursts or sustained fluxes, providing protection to sensitive optical components and devices against radiation-induced damage, which still remains a challenging problem. An optical limiter (OL) or optical power limiter (OPL) makes use of the nonlinear optical properties of materials to reduce significantly the transmittance of a device for high inputs. An ideal optical limiter has a linear transmittance at low input intensities, but above the threshold intensity its transmittance becomes constant.

The most significant application of an optical limiter is in the radiation protection of very sensitive optical components and sensors including human eye, from radiation-induced damage. They can be used to protect laboratory researchers from high-intensity laser radiation, safeguard military personnel from being blinded by the enemy light sources, or protect components in the devices that make use of laser light.

The selection of efficient materials is still the key point for optical power limiters and it has led to the study of materials that exhibit strong nonlinear absorption [2,3]. NLO organic molecules possess a strong donar-acceptor intermolecular interaction due to the presence of easily polarizable delocalized $\pi$-electrons in the system. Refractive nonlinearities associated with self-focusing and selfdefocusing in various media are currently being explored with greater interest owing to their increasing application potential in various optical devices, including optical power limiters [4].

The organic dye Eriochrome Cyanine R (ECR; LobaChemie, Mumbai ,India), belonging to triaryl methane family, is an important histological and bacterial stain and used for colouring textiles and leather. In this paper, we present the results of our investigations on the nonlinear OL response of the Organic Chromophore, ECR in aqueous solution under irradiation with $442 \mathrm{~nm} \mathrm{cw}$ He-Cd laser light for three different dopant concentrations. The OL behavior was also 
demonstrated. Z-scan experiments and nonlinear transmission measurements were carried out to investigate the OL behavior, of the dye Chromophore.

\section{EXPERIMENTAL}

The fundamental optical constants required for the analysis of the z-scan data of the samples were obtained from the optical absorption measurements recorded using a ultraviolet-visible (UV-Vis) spectrophotometer (Shimadzu UV-2450). The nonlinear transmission behavior of ECR dye were investigated using single-beam $\mathrm{Z}$-scan technique. The standard Z-scan set up [5], shown in Fig.1, was used for the measurement of NLO coefficient. The transmittance of the incident laser beam through an aperture in the far field was measured as a function of the sample position with respect to the focal plane. This formed a closed aperture scan. The experiment was performed at fixed input laser energy. The closed aperture scan is sensitive to nonlinear absorption as well as refraction[6]. OL experiments were also performed with ECR solution. The samples was kept at the focus of a converging lens (focal length $20 \mathrm{~cm}$ ) and the output was collected through a lens kept behind an aperture having $90 \%$ transmittance.

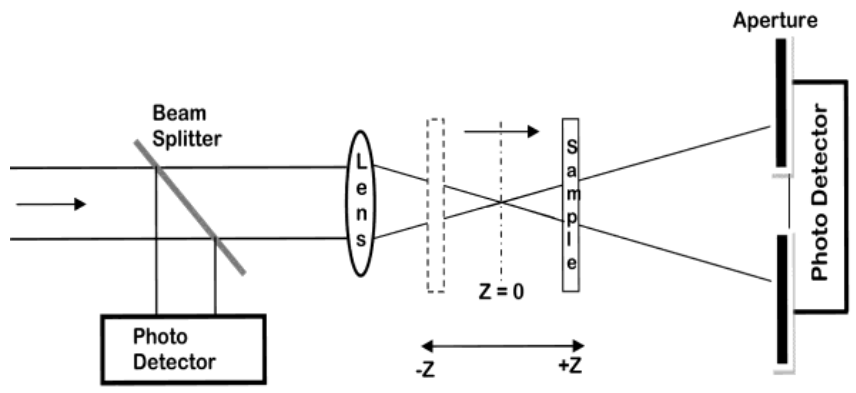

Figure 1: Schematic diagram of Z-scan experimental arrangement

\section{RESULTS AND DISCUSSION}

The linear absorption spectra of ECR dye in aqueous solution for various dye concentrations are shown in Fig:2. The standard value of absorption maxima for ECR dye is $518 \mathrm{~nm}$. The observed values of absorption maxima for the samples for different concentrations are $517 \mathrm{~nm}$. This shift in peak absorption wavelength is accounted for the structural and environmental changes occurring in the dye in polar solutions.

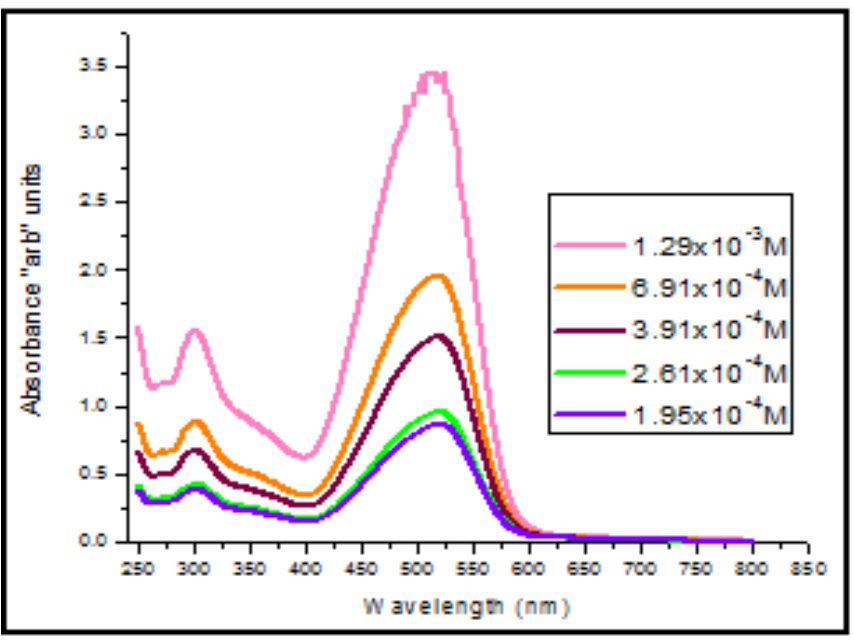

Figure 2: UV- Visible Absorption Spectra of Eriochrome Cyanine $\mathrm{R}$ in Solution

Optical limiting is an important NLO phenomenon, which can be utilized to protect delicate optical instruments, especially the human eye, from intense laser beams .An optical limiter should strongly attenuate intense, potentially dangerous laser beams, while exhibiting high transmittance for low intensity ambient light. Up to date, numerous inorganic and organic materials, such as), porphyrins, organic dyes, metal nanoclusters, quantum dots [11,12,13], etc. have been found to exhibit OL response.

Table 1: Output response of $\mathrm{EC} \mathrm{R}$ in aqueous solution having concentration $1.95 \times 10^{-4} \mathrm{M}$.

\begin{tabular}{|l|l|}
\hline Input power $(\mathrm{mW})$ & Output power $(\mathrm{mW})$ \\
\hline 57.6 & 21.52 \\
53 & 20.71 \\
42.3 & 14.88 \\
36.2 & 12.03 \\
20.79 & 6.14 \\
18.21 & 3.75 \\
18 & 3.66 \\
17.53 & 3.27 \\
12.49 & 1.48 \\
10.23 & .59 \\
\hline
\end{tabular}




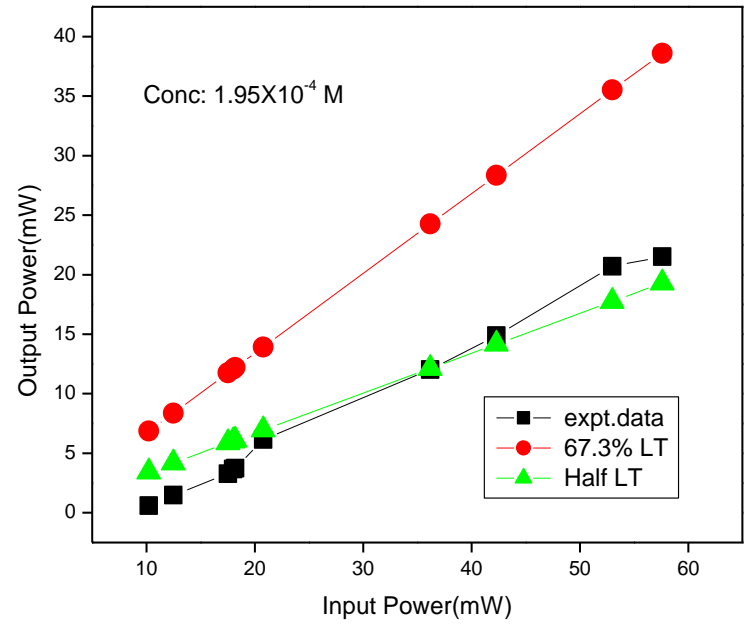

Fig :3 Optical Limiting behaviour of EC $\mathrm{R}$ in aqueous solution having concentration $1.95 \times 10^{-4} \mathrm{M}$.

With the extensive use of continuous wave lasers at power levels ranging from $\mathrm{mW}$ to $\mathrm{KW}$ for various applications, the need for protecting the human eye and the sensors used in handling the $\mathrm{CW}$ output has become increasingly important.

Optical Limiting based on nonlinear refraction is studied keeping an aperture $(2 \mathrm{~mm})$ in front of the detector. The aperture reduces the cross-section of the beam entering the power meter. Fig 3 shows the Optical Limiting performance of EC R in aqueous solution having concentration $1.95 \times 10^{-4}$ $\mathrm{M}$. The samples were placed at the valley position of the Zscan trace and the output power from the sample was monitored varying the input power in steps .

The OL response of EC R dye for $442 \mathrm{~nm} \mathrm{He}-\mathrm{Cd}$ light is demonstrated in Fig :3. The Straight line in figure represents the linear transmission [67.3\%]. It can be seen that at very low incident laser intensities, the transmission linearly increases, obeying Beer's law, and at high incident intensities the transmittance starts to deviate from linearity.

\section{CONCLUSION}

In summary, aqueous solutions of the organic dye EC R, were prepared for three different concentrations and its lowthreshold NLO properties were investigated. The effect of dye concentration on the absorption spectra was studied using UV-Visible spectrophotometer and found to be in well agreement with the Beer-Lambert law. The OL response of EC R dye for $442 \mathrm{~nm} \mathrm{He}-\mathrm{Cd}$ light is demonstrated in Fig :3 .The Straight line in figure represents the linear transmission [67.3\%]. It can be seen that at very low incident laser intensities, the transmission linearly increases, obeying
Beer's law, and at high incident intensities the transmittance starts to deviate from linearity.

\section{REFERENCES}

[1] Mathew George, C.I.Muneera, C.P.Singh, K.S.Bindra, S.M.Oak, "Z-scan studies and optical limiting of nanosecond laser pulses in neutral red dye", Optics \& Laser Technology 40(2008) 373-378.

[2] Xia T.Hagan DJ, Dogariu A, Said AA, Stryland EWV. Optimization of optical limiting devices based on excited-state absorption. Appl Opt 1997;36:4110-22

[3] BoggessTF ,Allan GR, Rychnovskysj, Labergerie DR, Venzke CH, Smirl AL, et al. Picrosecond investigations of optical limiting mechanisms in King's complex. Opt Eng 1993;32:1063-7

[4] G.Sree Kumar, B.ValsalaMilka ,C.I.Muneera, K.Sathiyamoorthy, C.Vijayan, "Nonlinear refraction and optical limiting in solid films of amido black dye under cw laser illumination", Optical Materials 30(2007) 311-313

[5] N.K.M.N.Srinivas, S.V.Rao, D.N.Rao. J. Opt. Soc.Am.B 20 (2003) 2470.

[6] Sheik-Bahae M, said AA, Wei TH, Wu YY, Hagan DJ, Stryland EWV. Sensitive measurement of optical nonlinearities using a single beam.IEEE J Quant Electron 1990;26:760-9

[7] Tripathy U and Bisht PB 2006 Opt. Commun. 261 353- 8

[8] C.Dou ,P.Wen ,X.Kong, S.Nakanishi, Q.Feng,Opt.Commn. 284(2011) 1067

[9] K.Sathiyamoorthy, C.Vijayan, M.Kothiyal,Opt.Matter.31(2008)79.

[10]American National Standard for safe use of Lasers ANSI Z136, 12000, www.laserinstitute.org.

[11]B.Clymer and S.A.Collins, Jr., Optical Engineering, 24,74 (1985)

[12]S.L. G'omez, F.L.S. Cuppo, and A.M. Figueiredo Neto "Nonlinear Optical Properties of Liquid Crystals Probed by Z-scan Technique" Brazilian Journal of Physics, vol. 33, no. 4, December, 2003

[13]Gu Bing and Wang Hui-Tian "Linear and Nonlinear Optical Properties of Ferroelectric Thin Films" School of Physics, Nankai University, China 